ing extreme events into hazards is surely to be naive. Bryant's conclusion that "when a natural disaster occurs . . . it is surprising that so few people die or are injured", also suggests that his optimism outweighs his judgement. Has he forgotten about the heavy death tolls in countries such as Bangladesh that have been caused by tropical storms and sea surges?

I cannot say that I share Bryant's optimism, not even for his book. Given the choice of texts on the subject, I would steer readers in other directions. $\square$

Allen H. Perry is in the Department of Geography, University College of Swansea, Swansea SA2 8PP, Wales, UK.

\section{Putting on the agony}

\section{A. W. Duggan}

Defeating Pain: The War Against a Silent Epidemic. By P. D. Wall and M. Jones. Plenum: 1991. Pp. 300. $\$ 24.95$.

The Culture of Pain. By David B. Morris. University of California Press: 1991. Pp. 375. \$29.95

Although a fair amount is known about how nerves signal damage and how the spinal cord processes this information, there is little understanding of how the brain handles the information to give pain perception. Any perception is associated with comparisons by the brain of past and present and it is axiomatic that cultural influences must be powerful in such comparisons. On this much, the authors of both these books are agreed. The approaches to pain taken by these two books, however, differ in many ways.

Wall and Jones set out to demystify pain. They are highly critical of the training of medical staff to see pain solely as an aid to diagnosis and not as a symptom requiring treatment in its own right. They argue that in many cases of intractable pain the cause either cannot be defined or cannot be eliminated and that, in these cases pain management should be made the top priority. In particular, repeated surgery purporting to remove causes of pain comes in for heavy criticism. The authors emphasize that the treatment of pain by simply attempting to interrupt a pathway from periphery to brain, either pharmacologically or surgically, is inadequate. Their main message for sufferers from chronic pain is that pain need not be tolerated and that sufferers should see themselves as active participants in its management.

Roughly 100 pages are devoted to

RADIO days - Advertisement for an RCA addon car radio. In the United States, the car radio became a familiar fixture in the 1930 s, with more than one million of them being sold in 1935 . This picture is taken from Michael Brian Schiffer's The Portable Radio in American Life, which traces the cultural and technological history of the appliance from the mid1890s, through the golden age in the 1920s, to the waning days of the "transistor revolution" in the 1960s. Published by the University of Arizona Press, price $\$ 45$.

explaining what is known about the scientific basis of nociception and pain. This section is liberally sprinkled with both clinical and everyday examples of pain and its paradoxes. Drugs used to treat pain are briefly dealt with; again the information to patients is not to fear drugs if they provide some relief. In an age when people are all too ready to condemn the use of pharmaceutical drugs, such advice is in itself a relief.

The other procedures used in pain management are so numerous that they are difficult to describe succinctly. Manipulation, nerve stimulation, acupuncture, surgical interventions, psychotherapy and the placebo effect all get due consideration.

Attempting to get a message across both to the general public and to health professionals is difficult, but in this task the authors of Defeating Pain can claim a large degree of success.

In contrast to Wall and Jones, Morris has few kind words to say about modern science. Indeed, he lays much of the blame for the epidemic of patients suffering from pain squarely at the feet of those who have taken an approach to pain based on anatomy, biochemistry and pharmacology and have neglected the cultural significance of pain. One of Morris's main conclusions is that pain would not be the cause of so much suffering and economic loss in Western society if its meaning in a cultural setting were appreciated by those who treat it. These are strong words, and although the author does call on many instances that illustrate how differing societies have viewed pain in differing contexts, I felt by the end of the book that this was an unproven hypothesis, albeit interesting.

Morris deals with the Christian approach to pain and suffering not only through early writings, but also through paintings and sculpture. The concept of Christian redemptive pain received a battering from the Marquis de Sade, and
Morris's interpretation of the writer's work as resulting from the mechanistic approaches of enlightenment medicine in the eighteenth century is skilfully developed.

The chapter on the "uses of pain" is rather disappointing, because Morris deals only with satire, torture and the use of pain to assert power and the pain of athletes and dancers. In fact, pain as a determinant of behaviour enabling an animal to survive is likely to be its main usefulness, but Morris is clearly employing the word "use" in the sense of a human inflicting pain or making a living through writing about pain.

In discussing tragedy as an art form, Morris seems happy to link suffering associated with pain perception with that from other causes. Suffering is difficult to define, but it may be regarded as a state resulting from the inability of an animal to interact with the environment in the accustomed (learned) way. Chronic pain is one cause of suffering, but so is isolation, starvation or immobilization. Whether the end result - appreciation of suffering in consciousness - is due to the same brain process is unknown, but (dare I say it) neuroscientists would like to think that ultimately that question can be answered.

The author's condemnation of what he calls the organic model of pain is rather overdone. To state that such thinking has been successful in silencing or subjugating all other cultural discourse on pain is nonsense.

Nonetheless, all those interested in pain would benefit from Morris's wide reading of both the general and scientific literature on the subject. $\mathrm{He}$ has many perceptive comments. Try not to be put off by his aversion to science. I, for one, thoroughly enjoyed reading the book.

A. W. Duggan is in the Department of Preclinical Veterinary Sciences, University of Edinburgh, Summerhall, Edinburgh EH9 1QH, UK. 